\title{
Introduction to the Third World Congress on Controversies to Consensus in Diabetes, Obesity and Hypertension (CODHy)
}

\section{Outcome studies versus clinical experience in the treatment of diabetes}

ITAMAR RAZ, MD ${ }^{1}$

Stefano Del Prato, mD ${ }^{2}$

$\mathbf{T}$ he recent increase in the number and variety of pharmacological agents for the treatment of diabetes and hypertension has created enormous challenges that in the past were unknown in clinical practice. These therapies aim to modify complex underlying pathologies, but do they ultimately promote health and prolong lives? The practicing clinician faces the daunting task of balancing the benefits of such therapies against their potential risks in the short and long term.

It is well known that along with reaching therapeutic targets and modifying pathologies, each therapy has side effects. One typical example is that of antidiabetes medications, which can lead to weight gain and hypoglycemia while requiring close monitoring that affects the patient's quality of life and raises the cost of treatment. Lately, the "art" of treating diabetes has presented physicians with particularly complex decisions concerning side effects. Studies published in the years 2007-2010 have shown that drugs can positively affect surrogate markers in the short term but prove to be damaging in the long term because of known as well as still unrecognized side effects $(1,2)$. In other words, a short-term positive response to therapy does not necessarily entail a positive outcome in the long term. As for new drugs, such as those from the incretin family, the risk of acute pancreatitis has not yet definitively been ruled out and these drugs have yet to be evaluated for other risks as well as cardiovascular safety.

Challenging decisions also concern the appropriate degree of modification for pathologies commonly associated with diabetes. Epidemiological studies have clearly demonstrated that obesity and hypertension, as well as fasting and, particularly, postprandial hyperglycemia, are associated with late micro- and macrovascular complications, hence the pursuit of near-normalization of these parameters. This indication may, however, not be valid for every diabetic patient, as recent trials have suggested attempting to normalize blood glucose or blood pressure with the full arsenal of existing drugs can sometimes be harmful $(3,4)$.

Last but not least, the effectiveness of each therapy, as well as its side effects, varies from one patient to another. Both are affected by such characteristics as the patient's age, duration of illness, damage to the target organs, and accompanying complications (5). In the face of such complexity, it is far from simple for the practitioner to feel convinced that a given

\footnotetext{
$\bullet \bullet \bullet \bullet \bullet \bullet \bullet \bullet \bullet \bullet \bullet \bullet \bullet \bullet \bullet \bullet \bullet \bullet \bullet \bullet \bullet \bullet \bullet \bullet \bullet \bullet \bullet \bullet \bullet \bullet \bullet \bullet \bullet \bullet \bullet \bullet \bullet \bullet \bullet \bullet \bullet \bullet \bullet \bullet \bullet \bullet$

From the ${ }^{1}$ Diabetes Unit, Hadassah University Hospital, Jerusalem, Israel; and the ${ }^{2}$ Department of Endocrinology and Metabolism, Section of Diabetes and Metabolic Diseases, University of Pisa, Pisa, Italy.

Corresponding author: Itamar Raz,ntv502@netvision.net.il.

This publication is based on the presentations at the 3rd World Congress on Controversies to Consensus in Diabetes, Obesity and Hypertension (CODHy). The Congress and the publication of this supplement were made possible in part by unrestricted educational grants from AstraZeneca, Boehringer Ingelheim, BristolMyers Squibb, Daiichi Sankyo, Eli Lilly, Ethicon Endo-Surgery, Generex Biotechnology, F. Hoffmann-La Roche, Janssen-Cilag, Johnson \& Johnson, Novo Nordisk, Medtronic, and Pfizer.

DOI: $10.2337 / \mathrm{dcl} 1-\mathrm{s} 201$

(C) 2011 by the American Diabetes Association. Readers may use this article as long as the work is properly cited, the use is educational and not for profit, and the work is not altered. See http://creativecommons.org/ licenses/by-nc-nd/3.0/ for details.
}

therapy will indeed ensure improved health-not to mention passing on this conviction to the patient, who is exposed to a wealth of medical information on the Internet and in the mass media.

The third World Congress on Controversies in Obesity, Diabetes and Hypertension (CODHy), held in Prague in May 2010, was intended to address the complexity inherent in today's treatment decisions. Its goal was to help practicing physicians integrate the numerous components offered by the wide variety of available therapies.

This supplement, divided into six sections, outlines the main controversies while attempting to present different sides to each debate. The idea is not to necessarily reach a consensus on therapeutic goals and choices, but rather to attempt to present the pros and cons of specific targets and therapies so that educated decisions can be made as dictated by each patient's individual characteristics.

The first section brings together articles under the heading "Diabetes, Obesity and Hypertension Outcome Studies." Such studies are of utmost importance because they might demonstrate that a drug's immediate impact on surrogate markers is not necessarily translated into reduced cardiovascular morbidity and mortality in the long term. The opening article in this section describes the new U.S. Food and Drug Administration's regulations for cardiovascular safety outcome studies, including the requirement that they be performed in Phases II and III of drug development and after the drug has been registered and commonly prescribed around the world. Other articles in this section provide the results of major recent outcome studies on diabetic patients. These studies have shown that aiming at more challenging targets, such as reducing blood glucose, lipids, and blood pressure to near-normal values, does not necessarily provide effective 
cardiovascular protection $(3,4)$ - a finding that calls for rethinking our approach to therapeutic targets and to the roles of different drugs in their attainment. The section ends with an article on obesity drugs, particularly sibutramine, found to increase the incidence of cardiovascular events in certain populations. We have included this discussion, even though sibutramine has been suspended from use, because it can teach us a great deal about the difficulties encountered in defining efficacy criteria for the development of new drugs.

The second section, "Diabetes and Cardiovascular Disease," provides an indepth discussion of cardiovascular risks associated with various aspects of diabetes. Do glucose variability, postprandial hyperglycemia, and hypoglycemia play a role in the development of cardiovascular disease? And, if so, does the modification of these pathologies lead to improved cardiovascular prognosis? In addition to addressing these controversial questions, this section deals with the potential association if not interaction between micro- and macrovascular complications. Another controversy discussed in the section concerns antiplatelet therapy: should it be administered to every patient with type 2 diabetes? The role of $\mathrm{C}$-reactive protein as a potential therapeutic target is also debated (6).

Diabetes in youth, rapidly becoming a problem of epidemic proportions worldwide, is the opening theme of the third section. Article topics include the prevention and treatment of type 2 diabetes in children, as well as the management of the metabolic syndrome and "double diabetes," which is more prevalent among pediatric patients. The section also deals with the pros and cons of using $\mathrm{HbA}_{1 \mathrm{c}}$ as a diagnostic tool for type 2 diabetes, and whether the target levels for $\mathrm{HbA}_{\mathrm{lc}}$ should be established on an individual basis. The pros and cons of continuous glucose monitoring are also discussed in view of the emerging connection between hypoglycemia and cardiovascular morbidity and mortality, which is widely reviewed in a separate article.

The fourth section deals with diabetes treatments and opens with a discussion of diabetes prevention: should it mainly rely on lifestyle interventions or should it resort to pharmacological means? Various controversies involved with diabetes therapy are also presented. The safety and place of peroxisome proliferator-activated receptor- $\gamma$ is discussed. As for the second-line therapies for diabetes after metformin, prominent candidates are insulin versus drugs from the incretin family. The section outlines the debate over the optimal timing of insulin therapy. It continues with an overview of multidrug therapy and the new and future drugs for the treatment of diabetes. Incretin therapy is reviewed in several articles. One of the articles discusses the incretin effect on $\beta$-cells and another debates whether incretins should be preferred over other classes of medications as a second-line therapy and which incretin is preferable: glucagon-like peptide-1 or dipeptidyl peptidase-4? The future and place of long-acting glucagon-like peptide-1s is also discussed.

In the fifth section, entitled "Hypertension," the focus is on the treatment of patients with multiple cardiovascular risk factors and end-organ damage. The section begins with several articles on the pathophysiology of hypertension and atherosclerosis in the diabetic patient and the role of the endothelium and inflammation in the development of the metabolic syndrome and diabetes complications.

The section then deals at length with the treatment of hypertension and kidney disease in diabetes. A major debate in this area, intensified by recent outcome studies, centers on the optimal level to which blood pressure should be reduced. What levels are to be considered normal in diabetic patients? Should the motto be "the lower the better?" One intriguing aspect of hypertension treatment concerns its "legacy" - the benefits that are thought to persist even after blood pressurelowering drugs are discontinued or stop being effective. The section ends with two topics: 1) the ongoing debate over prescribing (renin-angiotensin system) blockers to every diabetic patient, and 2) the relative significance of estimated glomerular filtration rate versus albuminuria as predictors for renal and cardiovascular outcomes in diabetes and the limitations of current therapy.

The sixth and final section, "Obesity/ Lipids," opens with a discussion of the relation of infection and vascular response to obesity and insulin resistance. The section follows with an article pointing out the difficulty of treating obesity, particularly in light of the limited number of drugs available for preventing or reducing this condition but elaborating on future drugs for obesity that are currently under development. The next two articles provide a critical analysis of bariatric surgery as a means of "partially curing" type 2 diabetes, including new approaches making such surgery applicable to patients with nonmorbid obesity.

Although the 2009 supplement dealt mainly with lipid deposits in the pancreas (where they cause $\beta$-cell dysfunction) and in the muscle (where they lead to insulin resistance), two articles in this supplement focus on lipids in other target organs, the liver and the heart, and the effect of these deposits on the patient's prognosis. The section ends with an article that debates statin versus combination therapy for the diabetic patient.

These articles intend to capture the very lively discussion that has characterized the CODHy meeting and to provide the reader with some consideration, some disagreement, and hopefully some consensus that may help in dealing with daily practice.

Acknowledgments-I.R. has served on the speakers bureau for Eli Lilly and Novo Nordisk; has served on the advisory board for Roche, Novo Nordisk, AstraZeneca, and Bristol-Myers Squibb; and has consulted for AstraZeneca/ Bristol-Myers Squibb and Andromeda and HealOr (local Israeli firms). No other potential conflicts of interest relevant to this article were reported.

\section{References}

1. Nissen SE, Wolski K. Effect of rosiglitazone on the risk of myocardial infarction and death from cardiovascular causes. N Engl J Med 2007;356:2457-2471

2. James WT, Caterson ID, Coutinho W, et al.; for the SCOUT Investigators. Effect of sibutramine on cardiovascular outcomes in overweight and obese subjects. N Engl J Med 2010;363:905-917

3. Gerstein HC, Miller ME, Byington RP, et al.; Action to Control Cardiovascular Risk in Diabetes Study Group. Effects of intensive glucose lowering in type 2 diabetes. $\mathrm{N}$ Engl J Med 2008;358:2545-2559

4. The ACCORD Study Group. Effects of intensive blood-pressure control in type 2 diabetes mellitus. N Engl J Med 2010;362: 1563-1574

5. Pozzilli P, Leslie RD, Chan J, et al. The AlC and ABCD of glycaemia management in type 2 diabetes: a physician's personalized approach. Diabetes Metab Res Rev 2010; 26:239-244

6. Ridker PM, Danielson E, Fonseca FA, et al.; JUPITER Study Group. Rosuvastatin to prevent vascular events in men and women with elevated C-reactive protein. N Engl J Med 2008;359:2195-2207 